\title{
Configurational Entropy Effects during Sorption of Hexane Isomers in Silicalite
}

\author{
S. Calero, B. Smit, and R. K rishna ${ }^{1}$ \\ D epartment of Chemical E ngineering, U niversity of A msterdam, N ieuwe A chtergracht 166, 1018 WV A msterdam, The N etherlands
}

R eceived M arch 20, 2001; revised M ay 23, 2001; accepted M ay 23, 2001

Santilli (J. Catal. 99, 335-341, 1986) has presented experimental data for sorption of hexane isomers, $n$-hexane $\left(n-C_{6}\right)$, 3-methylpentane (3MP), and 2,2-dimethylbutane (22D MB), in various zeolites. The experimental data on the loadings of the isomers at various temperatures using silicalite are particularly intriguing; 3MP and 22DMB are both seen to exhibit maxima in the sorption loadings with increasing temperature. We rationalise the experimental results by performing configurational-bias $\mathrm{M}$ onte $\mathrm{C}$ arlo simulations of sorption isotherms at varioustemperatures and pressures. While the linear $n-C_{6}$ can locate at either the straight or zigzag channels, configurational effects make the branched isomers locate only at the intersections. At a total mixture loading of four molecules per unit cell, all the intersections are fully occupied. Both branched isomers 3MP and 22DMB demand an extra "push" to locate within the channel interiors. Configurational entropy effects cause these branched isomers to be progressively "replaced" by $n$ $\mathrm{C}_{6}$ in order to achieve higher mixture loadings. The net result is that the branched isomers exhibit maxima at a total mixture loading of four molecules per unit cell. We discuss how configurational entropy effects can be exploited to separate hexane isomers with high selectivities. (c) 2001 A cademic Press

Key Words: configurational-bias Monte $\mathrm{C}$ arlo simulations; hexane isomers; ternary mixture.

\section{INTRODUCTION}

In the petroleum industry catalytic isomerisation is used to convert the linear alkanes to their branched isomers (1). I somerisation processes generate a mixture of isomers that usually require separation and recycle of the nonisomerised components. For example, the effluent of a paraffin isomerisation reactor may contain normal alkanes, mono-methyl alkanes, and di-methyl alkanes. Traditionally, only the normal alkanes would be separated from the mixture by adsorption and recycled to the isomerisation reactor, and any mono methyl alkanes would be collected with the di-methyl alkanes as product (2). However, it is the di-methyl alkanes that are the most desired and have the highest octane number

\footnotetext{
${ }^{1}$ To whom correspondence should be addressed. Fax: +31 205255604. E-mail: krishna@its.chem.uva.nl.
}

components. Consider for example the isomers of hexane: $\mathrm{n}$-hexane $\left(\mathrm{n}-\mathrm{C}_{6}\right)$ has a R O N (research octane number) $=30$ whereas the corresponding RON values for its isomers are 2-methylpentane ( $2 \mathrm{M} \mathrm{P})=74.5$; 3-methylpentane $(3 M P)=75.5 ; \quad 2,2-$ dimethylbutane $(22 \mathrm{DM} \mathrm{B})=94 ; \quad 2,3-$ dimethylbutane $(23 D M B)=105$. Therefore, the more efficient approach would be to adsorptively separate only the di-methyl alkanes as product and recycle the normal and the mono-methyl alkanes to the isomerisation reactor (3).

Consider the specific case of separating a mixture of $\mathrm{n}-\mathrm{C}_{6}, 3 \mathrm{MP}$, and 22D M B. The Henry coefficients of these isomers in, say, silicalite-1 are close to one another (4) and the separation factors are bel ow 3, which is too low for commercial exploitation. In this context, the experimental data reported in the paper by Santilli (5) are quite interesting. In a paper published in this journal, Santilli described a new experimental probe for measuring the concentration of molecules inside porous materials such as zeolites. To demonstrate the usefulness of his new probe he reported data on the sorption loadings of an equimolar mixture of $\mathrm{n}-\mathrm{C}_{6}, 3 \mathrm{MP}$, and 22D MB at a variety of temperatures in various zeolites. The data on the loadings reported for silicalite- 1 are particularly intriguing and have been replotted in Figs. $1 \mathrm{a}$ and $1 \mathrm{~b}$. The behaviour of $\mathrm{n}-\mathrm{C}_{6}$ is "normal," inasmuch as increasing temperature, at constant total pressure, results in a decrease in the sorption loading. The behaviours of $3 \mathrm{M} \mathrm{P}$ and $22 \mathrm{D} \mathrm{M} \mathrm{B}$, in sharp contrast, are quite "abnormal;" these branched isomers initially show an increase in the loading with increasing temperature until a maximum is reached and beyond this point the loadings decrease with increasing temperature. In Fig. $1 \mathrm{c}$ the $n-\mathrm{C}_{6} / 3 \mathrm{M} \mathrm{P}$ and $n-C_{6} / 22 D M B$ selectivities, defined as the ratio of the loadings, are plotted against the temperature. We note that the selectivities approach unity at high temperatures and increase significantly as the temperature is reduced to below $400 \mathrm{~K}$.

Curiously, Santilli (5) does not even comment on these data, and focuses his discussions on demonstrating the efficacy of his measurement probe! We aim to show in this paper that the Santilli data are of practical significance in the context of the development of improved sorption based separations of alkanes isomers. We begin by attempting to 
(a) nC6\& total mixture loadings

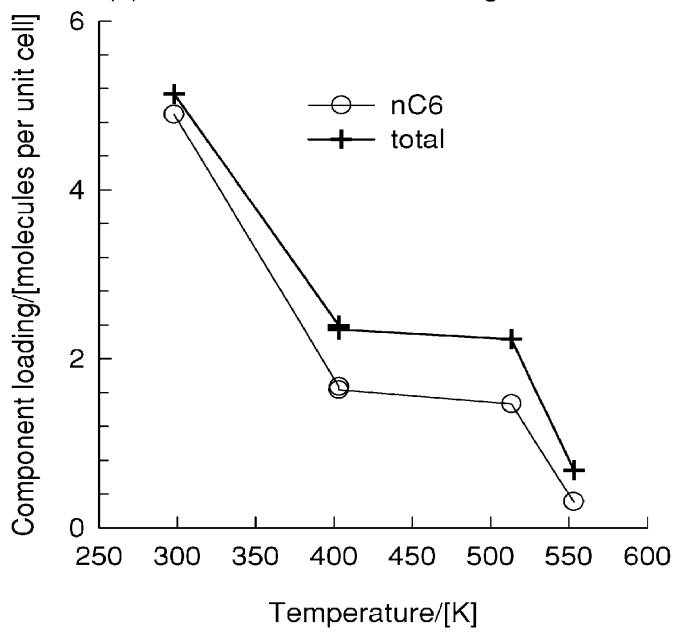

(b) 3MP \& 22DMB loadings

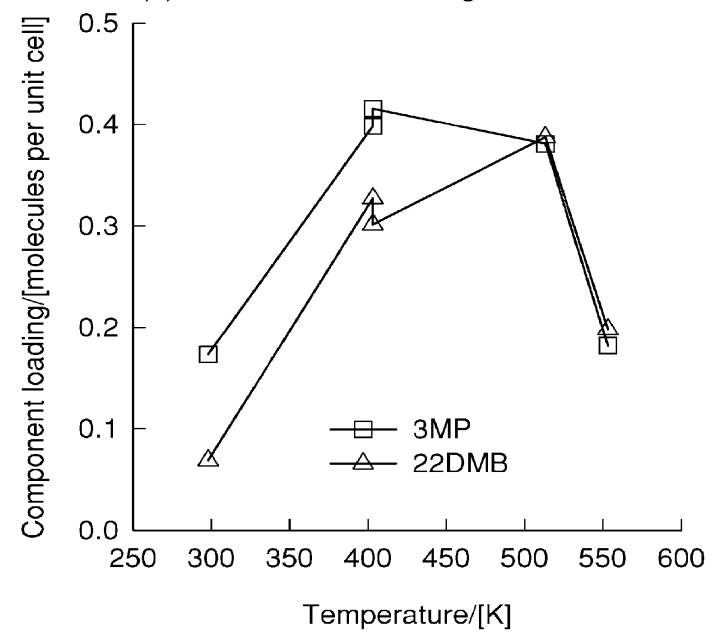

(c) nC6/3MP \& nC6/22DMB selectivities

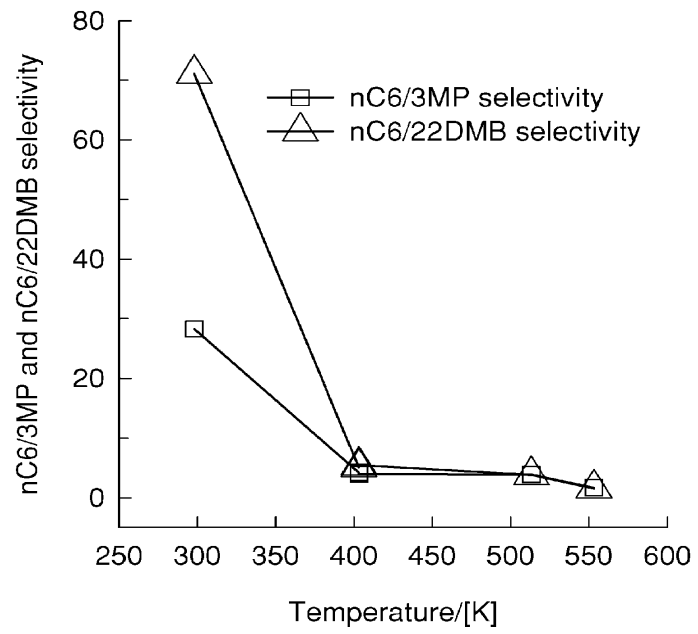

FIG. 1. ( $a$ and $b$ ) Component loadings for an equimolar mixture of $\mathrm{n}-\mathrm{C}_{6}, 3 \mathrm{M} \mathrm{P}$, and 22D M B as a function of temperature. (c) Sorption selectivity as a function of temperature. Experimental data at constant pressure from Santilli (5). rationalise the experimental results of Santilli by performing configurational-bias $\mathrm{M}$ onte $\mathrm{C}$ arlo (CBM C) simulations of the sorption isotherms for pure components, three binary mixtures, and the ternary mixture in silicalite. We had used the CBMC simulation technique in earlier publications to study single and binary sorption isotherms of alkanes (6-8); the computational details are to be found in R ef. (7). We have extended the CBMC technique to calculate ternary sorption isotherms, for which the number of M onte Carlo cycles was increased to $1 \times 10^{8}$.

\section{CONFIGURATIONAL-BIAS MONTE CARLO (CBMC) SIMULATION TECHNIQUE}

The linear and branched alkanes are described with a united-atom model, in which $\mathrm{CH}_{3}, \mathrm{CH}_{2}$, and $\mathrm{CH}$ groups are considered as single interaction centres. When these pseudoatoms belong to different molecules or to the same molecule but separated by more than three bonds, the interaction is given by a L ennard-J ones potential. The bonded interactions include bond-bending and torsion potentials. We consider the zeolite lattice to be rigid and we assume that interactions of an alkane with the zeolite are dominated by the dispersive forces between alkane pseudoatoms and the oxygen atoms of the zeolite. These interactions are described by a Lennard-J ones potential, with parameters given in $\mathrm{R}$ ef. (7).

A dsorption isotherms are computed using a $\mathrm{M}$ onte $\mathrm{C}$ arlo simulation in the grand canonical ensemble. In this ensemble the temperature and chemical potentials are imposed. The average number of adsorbed molecules per unit cell of the zeolite follows from the simulations. Characteristic of these types of simulations is that during the calculations attempts are made to change the total number of particles by making attempts to insert a molecule into or remove a molecule from the zeolite. To make these types of moves possible for the long-chain alkanes, we use the configurational-bias M onte Carlo (CBMC) technique. Instead of inserting a molecule at a random position, in a CBMC simulation a molecule is grown atom by atom in such a way that the "empty spots" in the zeolite are found. This growing scheme gives a bias that is removed exactly by adjusting the acceptance rules (7). CBM C simulations were performed in cycles and in each cycle, an attempt to perform one of the following moves was made:

(1) D isplacement of a randomly selected chain. The maximum displacement was adjusted to an overall acceptance of $50 \%$.

(2) R otation of a chain around its centre of mass. The chain is chosen at random and the maximum rotation angle is selected to accept $50 \%$ of the moves.

(3) Partly regrowing of a chain; a chain is selected at random and part of the molecule is regrown using the CBM C 
scheme. It is decided at random which part of the chain is regrown and with which segment the regrowing is started.

(4) Exchange with a reservoir. It is decided at random whether to add or to remove a molecule from the zeolite following the acceptance rules derived by $\mathrm{V}$ lugt et al. (7).

(5) Change of identity (only in the case of mixtures). O ne of the components is selected at random and an attempt is made to change its identity.

The numbers of Monte Carlo cycles performed for one-component, two-component, and three-component isotherms were, respectively, $2 \times 10^{7}, 5 \times 10^{7}$, and $1 \times 10^{8}$. The relative probabilities of moves were $15 \%$ displacements, $15 \%$ rotations, $15 \%$ partial regrowths, and $50 \%$ exchanges with the reservoir, and the remaining $5 \%$ of the moves were attempts to change the identity of a molecule.

\section{SIMULATION RESULTS AND DISCUSSION}

B efore mixture effects are considered let us examine the pure component sorption isotherms calculated using CB M C techniques at $362 \mathrm{~K}$, a temperature within the range studied by Santilli (5); the pure component isotherms are shown in Fig. 2. We see that $n-C_{6}$ shows a slight inflection at a molecular loading $\Theta=4$ due to commensurate freezing (9). The branched isomer 3M P shows a pronounced inflection at $\Theta=4$ because these molecules prefer to locate at the intersections between the straight and zig-zag channels of silicalite; to push them into the channel interiors requires an extra "push," leading to inflection behaviour. The isomer 22D M B is so bulky that it can be located only at the intersections; there is no inflection for this component.

The sorption isotherms for 50-50 mixtures of the three binary pairs are shown in Fig. 3. The $n-C_{6}-3 M P$ and $3 M P-$ 22D M B binaries have been determined at $362 \mathrm{~K}$ and the

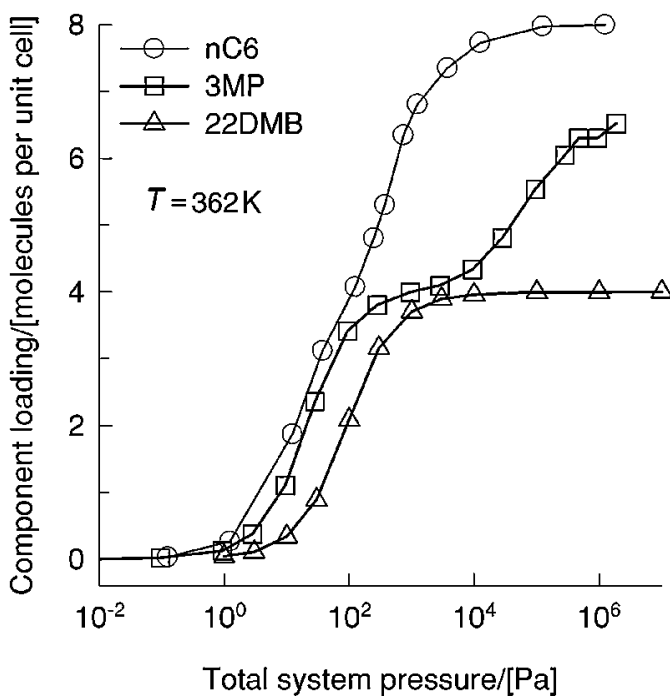

FIG. 2. Pure component isotherms for $n-C_{6}, 3 M P$, and $22 D M B$ at $362 \mathrm{~K}$ obtained from CBM C calculations.
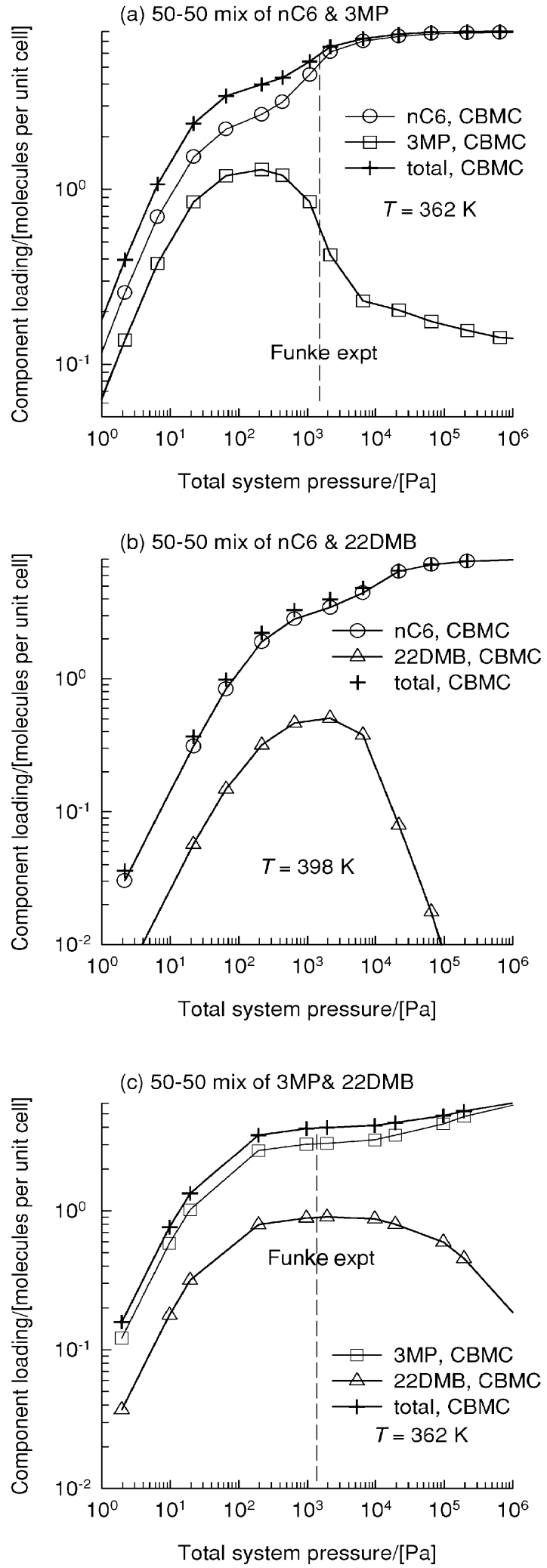

FIG. 3. (a) CBMC simulations of loadings for 50-50 mixtures of (a) $n-C_{6}-3 M P$ at $362 K$, (b) $n-C_{6}-22 D M B$ at $398 K$, and (c) $3 M P-22 D M B$ at $362 \mathrm{~K}$ for various pressures. 
$n-C_{6}-22 D M B$ binary at $398 \mathrm{~K}$, in order to allow comparison with the membrane permeation data of F unke etal. (10) and Gump et al. (11).

For the binary mixture of $n-C_{6}$ and $3 M P$ at $362 \mathrm{~K}$ (see Fig. 3a), it is interesting to note the maximum in the loading of $3 \mathrm{M} \mathrm{P}$ when the total mixture loading is precisely four molecules per unit cell, when the intersections are all occupied; this occurs at a total pressure of about $100 \mathrm{~Pa}$. When the pressure is raised above $100 \mathrm{~Pa}$ the loading of 3M P reduces virtually to zero. The $n-C_{6}$ has a higher packing efficiency within the silicalite matrix than the 3M P molecules. It is more efficient to obtain higher loading by "replacing" the $3 \mathrm{M} P$ with $\mathrm{n}-\mathrm{C}_{6}$; this configurational entropy effect is the reason behind the curious maxima in the $3 \mathrm{M} P$ loading in the mixture.

Funke et al. (10) measured the permeation selectivities for 50-50 mixtures of $n-C_{6}$ and $3 M P$ at $362 K$, keeping the upstream hydrocarbons pressure at $15 \mathrm{kPa}$; see Table 3 of their paper. The permeation selectivity, defined as the ratio of permeation fluxes of the linear and branched alkanes, is proportional to the ratio of the loadings of the two components, i.e., the sorption selectivity, at the upstream face of the membrane. Funke et al. (10) observed that the permeation selectivity was 24 , which was much higher than the permeation selectivity obtained on the basis of pure component permeation data, i.e., 1.3. This high permeation selectivity can be explained by examination of Fig. 3a, where the upstream pressure ( $15 \mathrm{kPa}$ ) condition of the Funke experiment is indicated by the dashed vertical line. The upstream pressure corresponds to a situation well beyond the pressure at which the $3 \mathrm{MP}$ loading exhibits a maximum. A t this upstream pressure of $15 \mathrm{kPa}$ the sorption selectivity is significantly higher than that at upstream pressures below $100 \mathrm{~Pa}$. Funke et al. (10) also report permeation selectivities for 50-50 mixtures at four different temperatures, keeping the upstream hydrocarbons pressure at $15 \mathrm{kPa}$; see Fig. 4. We performed CB M C simulations at various temperatures keeping the total hydrocarbons pressure at $15 \mathrm{kPa}$ and have compared the ratio of the sorption loadings of $\mathrm{n}-\mathrm{C}_{6}$ and $3 \mathrm{MP}$ with the permeation selectivity; see Fig. 4. We note that the permeation and sorption selectivities show the same trend with temperature and are in good agreement with each another. The increase in the $n-C_{6} / 3 M P$ selectivity with decreasing temperature is to be attributed to the fact that the total mixture loading increases to values in excess of four molecules per unit cell when all the intersections are occupied; this occurs at a temperature of $400 \mathrm{~K}$. F urthermore, we note that the temperature dependence of the $\mathrm{n}-\mathrm{C}_{6} / 3 \mathrm{M} \mathrm{P}$ selectivity follows the same trend as that seen in Fig. 1c for the Santilli data.

Consider now the 50-50 binary mixture of $\mathrm{n}-\mathrm{C}_{6}$ and 22D MB at $398 \mathrm{~K}$; see Fig. 3b. The di-branched isomer 22D M B shows a pronounced maximum when the total mixture loading is 4; this occurs at a total hydrocarbons pres-

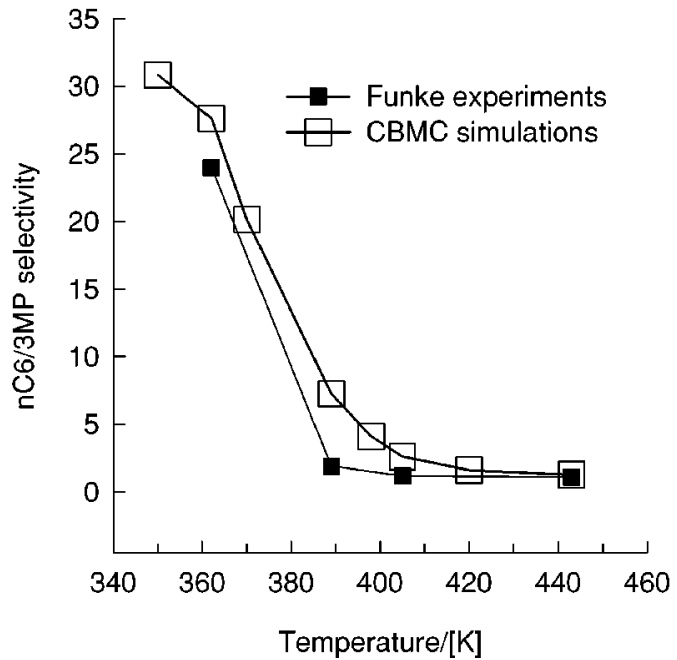

FIG . 4. $n-C_{6} / 3 M P$ selectivity for 50-50 mixture permeation across silicalite membrane. The experimental data of Funke etal. (10) are compared with CBM C simulations at various temperatures. The total hydrocarbons pressure is maintained at $15 \mathrm{kPa}$.

sure of 2000 Pa. G ump et al. (11) have reported the permeation fluxes of 50-50 mixtures of $n-\mathrm{C}_{6}$ and 22D M B across a silicalite membrane at $398 \mathrm{~K}$ for various upstream hydrocarbon pressures; see Figs. 5 and 6 of their paper. Since the flux of any component is proportional to the loading at the upstream face, we would expect the flux of 22D M B to go through a maximum as the upstream compartment pressure is increased, in steps, from say $100 \mathrm{~Pa}$ to $100 \mathrm{kPa}$. This is precisely what G ump et al. (11) have observed in their experiments. The experimental fluxes of 22D M B are compared in Fig. 5 with the 22D M B loadings obtained from

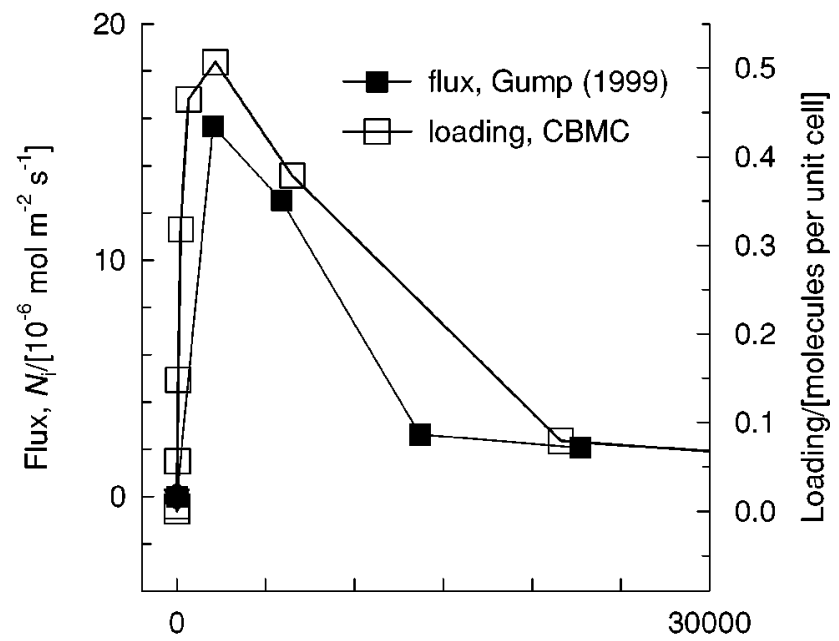

Total hydrocarbons pressure/[Pa]

FIG . 5. Flux of 22D M B during permeation of a 50-50 mixture of $n-C_{6}$ and 22D M B across silicalite membrane at $398 \mathrm{~K}$, measured by $\mathrm{G}$ ump et al. (11), compared with 22D M B loading obtained from CB M C simulations. 
(a) Component loadings vs total pressure

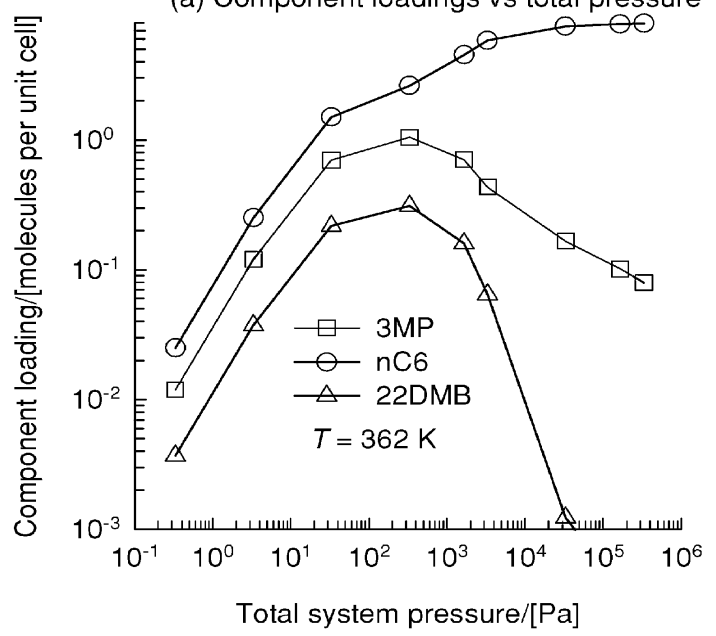

(b) Component loadings vs total loading

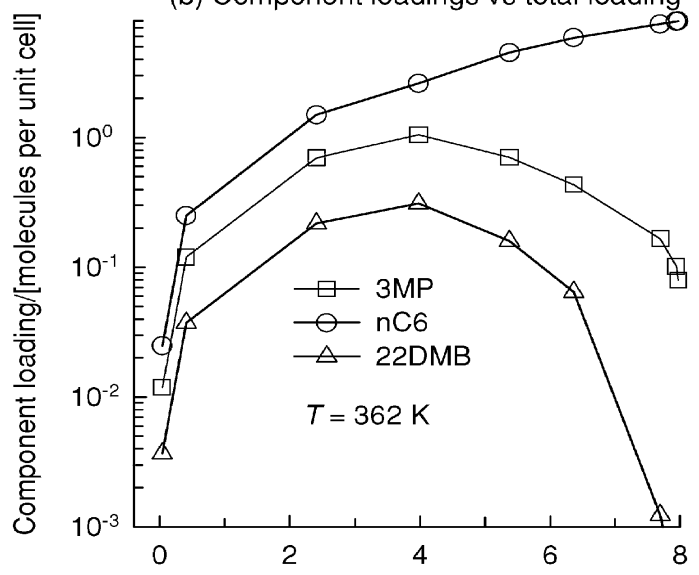

Total loading/[molecules per unit cell]

(c) Sorption selectivity vs total loading

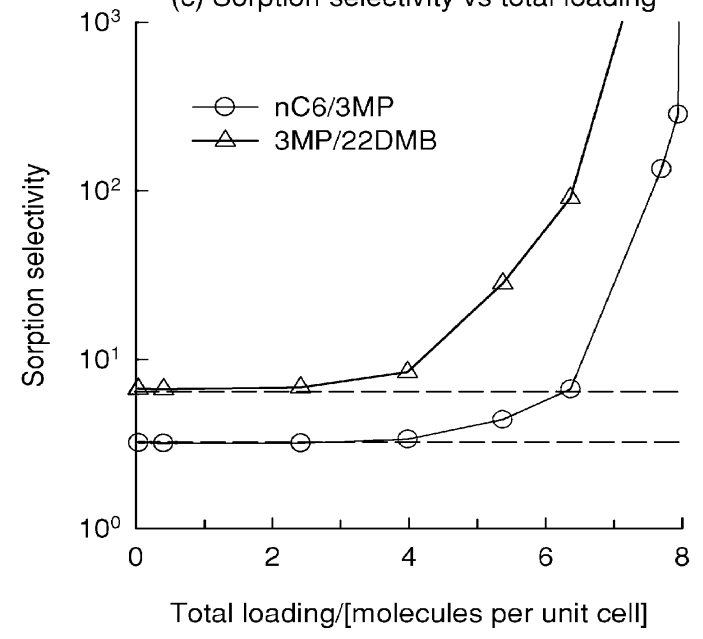

FIG. 6. (a) CBMC simulations of loadings for equimolar mixture of $\mathrm{n}-\mathrm{C}_{6}-3 \mathrm{M}$ P-22DM B at $362 \mathrm{~K}$. (b) Component loadings vs total mixture loading. (c) Sorption selectivities as a function of total loading.
CBM C simulations. It is heartening to note that the experimentally observed maximum flux of 22D M B is obtained at the same pressure at which the 22D M B exhibits a maximum in its loading, i.e., $2000 \mathrm{~Pa}$.

Let us now consider sorption of a 50-50 mixture of 3M $P$ and $22 \mathrm{D} \mathrm{M} \mathrm{B}$ at $362 \mathrm{~K}$ (see Fig. 3c); both 3M P and 22D M B prefer to locate themselves at the intersections, which are roomy enough to accommodate the side chains. H owever, at a total loading $\Theta=4$, the intersections are all fully occupied; this occurs at a total pressure of $1 \mathrm{kPa}$. Increasing the pressure from 1 to $10 \mathrm{kPa}$ has practically no influence of the loadings of $3 \mathrm{M} \mathrm{P}, 22 \mathrm{D} \mathrm{M} \mathrm{B}$, and of the total mixture. A $\mathrm{t}$ pressures in excess of $20 \mathrm{kPa}$, it is possible to "push" 3M P into the channel interiors. Hence, the $3 \mathrm{MP}$ loading and that of the total mixture increase. However, the 22D M B molecules are too bulky to be located in locations other than at the intersections. I nterestingly, we note that at pressures in excess of $2 \mathrm{kPa}$, the 22D M B loadings decrease with increasing pressure. A pparently it is energetically more efficient for the silicalite matrix to fill itself with $3 \mathrm{MP}$ and "throw" out the stubborn 22D M B molecules. Configurational entropy effects are the reason behind the "table mountain" maximum in the loading of 22D M B. A t sufficiently high pressures, not of technological relevance, the 22D M B loading in the mixture would reduce to practically zero.

Funke et al. (10) have also reported the permeation selectivities for a 50-50 mixture of 3M P and 22D M B across a silicalite membrane at $362 \mathrm{~K}$, keeping the upstream hydrocarbons pressure at $15 \mathrm{kPa}$; see Table 5 of their paper. A t a pressure of $15 \mathrm{kPa}$, the sorption selectivities are expected to be low and Funke et al. (10) report permeation selectivities of unity, i.e., practically no separation of the two isomers. $\mathrm{H}$ ad their experiments been carried out with an upstream hydrocarbons pressure in excess of $100 \mathrm{kPa}$, they would have obtained much higher selectivities. Since at such high pressures, the hydrocarbon mixture would be in the liquid phase, one technological solution would be to operate in the pervaporation mode (upstream compartment in the liquid phase; downstream compartment in the vapour phase). M atsufuji et al. (12) have shown that high selectivities for the separation of hexane isomers can be obtained by operating in the pervaporation mode, underlining our arguments.

$\mathrm{H}$ aving underlined the influence of configurational entropy effects during sorption of the binary pairs of hexane isomers, we consider the importance of these effects in a ternary mixture. CBM C simulations for an equimolar ternary mixture of $\mathrm{n}-\mathrm{C}_{6}, 3 \mathrm{MP}$, and 22D M B at $362 \mathrm{~K}$ are shown in Fig. 6a for a range of total pressures. The loadings of the three components follow "normal" behaviour for pressures below $50 \mathrm{~Pa}$ and the sorption loadings follow the hierarchy dictated by the H enry coefficients. A t a pressure of $500 \mathrm{~Pa}$, the total mixture loading is four molecules 
per unit cell; at this loading all the intersection sites are fully occupied. A t pressures exceeding $500 \mathrm{~Pa}$, the silicalite matrix prefers to accept $n-C_{6}$ into its matrix rather than either of the branched isomers. The loadings of both $3 \mathrm{M} \mathrm{P}$ and 22D M B decrease as the pressure is increased beyond $500 \mathrm{~Pa}$. There are three types of entropic "battles" in play at pressures exceeding $500 \mathrm{~Pa}$. The battle between linear isomer and its branched isomers is won by the linear isomer. The battle between the mono-methyl and dimethyl isomers is won by the mono-methyl isomer.

A more useful way to examine the ternary sorption data is to plot the component loadings against the total mixture

(a) nC6/3MP selectivity

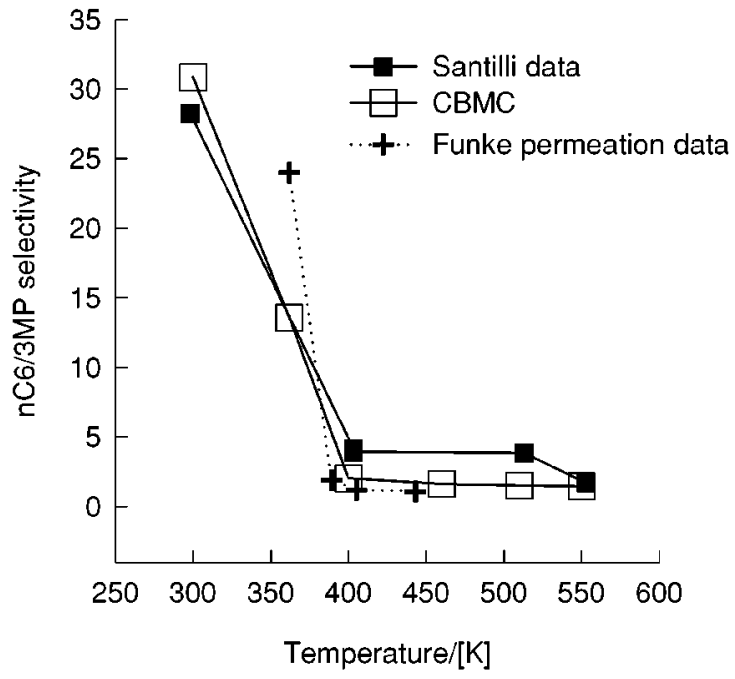

(b) $\mathrm{nC6} / 22 \mathrm{DMB}$ selectivity

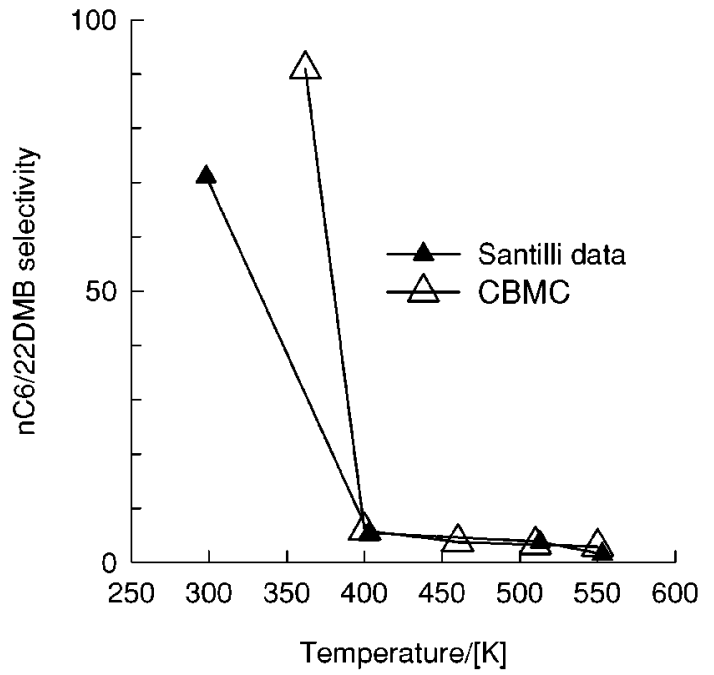

FIG . 7. Comparison of sorption selectivity for (a) $n-C_{6} / 3 M P$ and (b) $n-C_{6} / 22 D M B$ in equimolar ternary mixtures. The experimental data of Santilli (5) are compared with calculations from CBM C simulations. The CBM C simulations have been carried out at a total pressure of $3 \mathrm{kPa}$. A Iso shown in (a) are the permeation $n-C_{6} / 3 M P$ selectivities obtained by $F$ unke et al. (10).

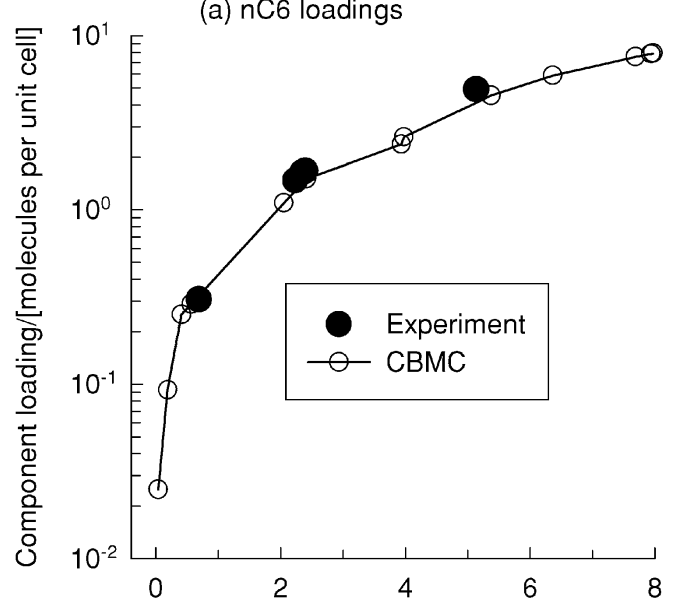

Total mixture loading / [molecules per unit cell]

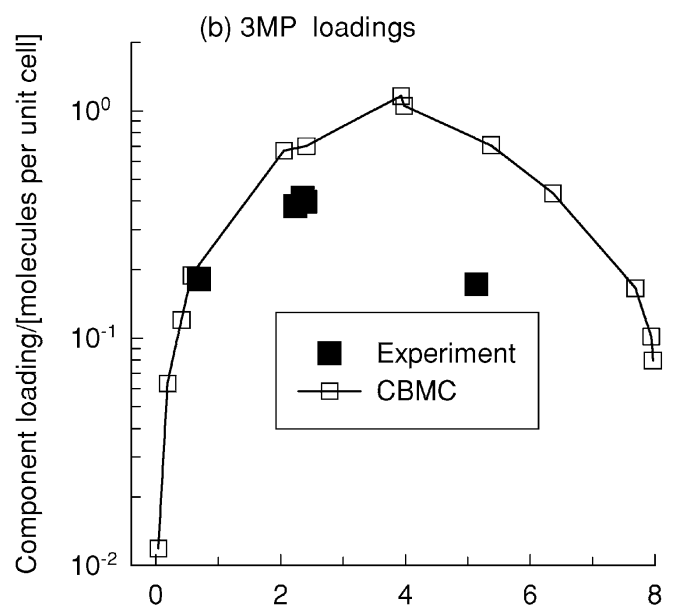

Total mixture loading /[molecules per unit cell]

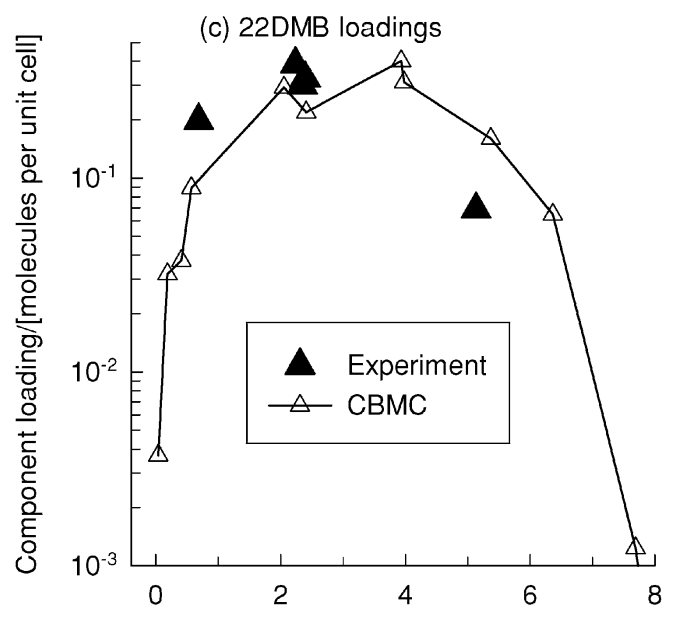

Total mixture loading /[molecules per unit cell]

FIG. 8. Component loadings for an equimolar mixture of $n-C_{6}-3 M P-$ 22DMB as a function of total mixture loading. Experimental data of Santilli (5) are compared with CB M C simulations. The CB M C simulations were carried out at (i) $T=300,362,400,460,510,550 \mathrm{~K}$ and pressure $=$ $3 \mathrm{kPa}$ and (ii) $T=362 \mathrm{~K}$ and a variety of pressures. 
loading; see Fig. $6 \mathrm{~b}$. We clearly note the maxima in the $3 \mathrm{M} \mathrm{P}$ and 22D M B loadings when the total mixture loading equals 4. The $-C_{6} / 3 M P$ and $3 M P / 22 D M B$ sorption selectivities increase significantly when the total mixture loading exceeds 4; see Fig. 6c.

In order to rationalise the Santilli data shown in Fig. 1, we performed a series of simulations at constant total pressure of $3 \mathrm{kPa}$ and temperatures of $300,362,400,460,510$ and $550 \mathrm{~K}$. Figure $7 \mathrm{a}$ compares the $\mathrm{n}-\mathrm{C}_{6} / 3 \mathrm{MP}$ selectivity obtained from the CBMC simulations with those reported by Santilli. A Iso plotted in Fig. 7a are the permeation selectivities reported by Funke et al. (10). The three sets of selectivities are in agreement with one other. We therefore may conclude that the increase in selectivity with decreasing temperature is due to the fact that the mixture loading increases to values significantly higher than 4 when the temperature is decreased below $400 \mathrm{~K}$. The Santilli data (5) provide a direct confirmation of the concept of configurational entropy affecting mixture sorption and lend credence to our $\mathrm{CBMC}$ simulations.

In Fig. $7 \mathrm{~b}$ the $\mathrm{n}-\mathrm{C}_{6} / 22 \mathrm{D} M$ B sorption selectivity obtained from CBMC simulations is compared with the data of Santilli (5); again good agreement is obtained. The strong increase in the selectivity when the temperature is reduced below $400 \mathrm{~K}$ is again to be attributed to strong configurational entropy effects which favour the linear molecule.

For direct comparison of the Santilli data with CBM C simulations, we plot the component loadings of the three hexane isomers as a function of the total mixture loadings in Figs. $8 \mathrm{a}, 8 \mathrm{~b}$ and $8 \mathrm{c}$. A Iso shown in Fig. 8 are CB M C simulation results obtained at (i) $T=300,362,400,460,510,550 \mathrm{~K}$ and pressure $=3 \mathrm{kPa}$ and (ii) $T=362 \mathrm{~K}$ and a variety of pressures. Figure 8a shows good agreement between the experimentally determined $\mathrm{n}-\mathrm{C}_{6}$ loadings and those calculated from CBM C.In Figs. $8 \mathrm{~b}$ and $8 \mathrm{c}$ we note that the CB M C simulations predict maxima in the $3 \mathrm{MP}$ and $22 \mathrm{D} \mathrm{M} \mathrm{B} \mathrm{load-}$ ings when the total mixture loading is four molecules per unit cell. U nfortunately, the Santilli data are available only for total loadings of two and six molecules per unit cell. N evertheless, the experiments confirm that there is a maximum in the loading of the branched alkane, 3M P and 22D M B at a mixture loading between two and six moleculesper unit cell.

The results shown in Fig. 8 have practical implications. Provided the mixture loading within silicalite is higher than four molecules per unit cell, the sorption hierarchy is as follows: $n-C_{6} \gg 3 M P \gg 22 D M B$, with the di-branched alkane being virtually excluded from the zeolite. It appears from the patent of $D$ andekar et al. (3) that the entropy-based sep- aration concept is recognised by industry and that a combined sorption-reaction process is being developed relying on this separation principle.

\section{CONCLUDING REMARKS}

We have rationalised the "curious" experimental data of Santilli (5) by performing CBM C simulations. Configurational entropy effects come into play when the total mixture loading is higher than four molecules per unit cell and if these conditions are maintained we obtain the sorption hierarchy $n-C_{6} \gg 3 M P \gg 22 D M B$, with the di-branched alkane being virtually excluded from the zeolite. Further support of the Santilli data is provided by the permeation data of Funke et al. (10) and Gump et al. (11) for 50-50 mixtures of $n-C_{6}-3 M P$ and $n-C_{6}-22 D M B$ across a silicalite membrane. The entropy-based sorption principle has commercial potential, which has been recognised by industry (3); our paper provides the scientific background for the separation concept.

\section{ACKN OWLEDGMENTS}

B.S. and R.K. have received grants from the $\mathrm{N}$ etherlands $\mathrm{O}$ rganisation for Scientific R esearch (NWO), through the $N$ etherlands R esearch Council for Chemical Sciences (CW).

\section{REFERENCES}

1. Chen, N. Y., D egnan, T. F., and Smith, C. M., "M olecular transport and reaction in zeolites. Design and application of shape selective catalysts." V CH, N ew Y ork, 1994.

2. H aizmann, R . S., H ibbs, F. M ., and R aghuram, S., U S 5043525 to U O P, Inc., 1993.

3. D andekar, H. W., Funk, G . A ., and Z innen, H . A ., U S 6069289 to U O P, Inc., 2000.

4. D enayer, J. F., Baron, G. V., M artens, J. A ., and Jacobs, P. A ., J. Phys. Chem. B 102, 3077 (1998).

5. Santilli, D. S., J. Catal. 99, 335 (1986).

6. Krishna, R., Smit, B., and V lugt, T. J. H., J. Phys. Chem. A 102, 7727 (1998).

7. V lugt, T. J. H., K rishna, R ., and Smit, B., J. Phys. Chem. B 103, 1102 (1999).

8. Schenk, M., Vidal, S. L., V lugt, T. J. H., Smit, B., and K rishna, R., L angmuir 17, 1558 (2001).

9. Smit, B., and M aesen, T. L. M ., N ature 374, 42 (1995).

10. Funke, H. H., A rgo, A . M ., Falconer, J. L ., and N oble, R. M ., Ind. Eng. Chem. Res. 36, 137 (1997).

11. Gump, C. J., Noble, R. D., and Falconer, J. L., Ind. Eng. Chem. Res. 38, 2775 (1999).

12. M atsufuji, T., Watanabe, $K$., N ishiyama, N., E gashira, $Y$., M atsukata, M., and U eyama, K., Ind. E ng. Chem. 39, 2434 (2000). 